\title{
New Onset Intraparenchymal Hemorrhage Following Infant Feeding Tube-Guided Evacuation of Chronic Subdural Hematoma: A Report of Two Cases
}

\author{
${ }^{1}$ Department of Neuroanesthesiology and Critical Care, All India \\ Institute of Medical Sciences, New Delhi, India \\ ${ }^{2}$ Department of Neurosurgery, All India Institute of Medical \\ Sciences, New Delhi, India
}

Ankur Khandelwal ${ }^{1}$ Gyaninder P. Singh ${ }^{1}$ Mayank Garg ${ }^{2} \quad$ Arvind Chaturvedi ${ }^{1}$

\begin{abstract}
Address for correspondence Gyaninder P. Singh, MD, DM, Department of Neuroanaesthesiology and Critical Care, All India Institute of Medical Sciences, Neurosciences Centre, Room No. 711 (7th Floor), New Delhi 110029, India (e-mail: drsingh_gp@yahoo.co.in).
\end{abstract}

J Neuroanaesthesiol Crit Care:2021;8:63-65

\begin{abstract}
Keywords

- chronic subdural hematoma

- infant feeding tube

- intraparenchymal hemorrhage

Chronic subdural hematoma (CSDH) is one of the most common neurosurgical conditions and is usually treated by simple burr-hole drainage. Prognosis is usually good unless complications occur. Use of infant feeding tube (IFT) for irrigation of hematoma in case of CSDH with septum is a common practice. However, it poses a significant risk. We present two cases of intraparenchymal hemorrhage as a complication of CSDH evacuation in which IFT was used for irrigation.
\end{abstract}

\section{Introduction}

Chronic subdural hematoma (CSDH) is a common pathology in neurosurgical practice and is defined as hematoma with duration of more than 3 weeks. ${ }^{1}$ Surgical management with burr-hole evacuation of symptomatic SDH is the standard method of treatment. During burr-hole technique for CSDH evacuation, infant feeding tube (IFT) is commonly used as a channel to irrigate the subdural cavity with septum. The advantages include low cost and easy availability. However, even though rare, IFT-guided evacuation of CSDH might be hazardous. We experienced two cases of intraparenchymal hemorrhage (IPH) in which IFT was used as an irrigating channel for the evacuation of residual blood of CSDH.

\section{Case 1}

A 95-year-old female patient, diagnosed with right frontotemporoparietal (FTP) CSDH ( $\bullet$ Fig. 1A) and having Glasgow Coma Score (GCS) of 13/15 (E3V4M6), was posted for burr-hole and hematoma evacuation. The anesthetic management included intravenous administration of midazolam (1 mg) and fentanyl $(50 \mu \mathrm{g})$ followed by bilateral scalp block using bupivacaine $0.25 \% \mathrm{w} / \mathrm{v}$ solution. Oxygen was administered through face mask at $5 \mathrm{~L} / \mathrm{min}$. The head was allowed to rest on horse-shoe head rest. A baseline arterial blood gas (ABG) analysis showed $\mathrm{pH} 7.40, \mathrm{PCO}_{2} 37 \mathrm{~mm} \mathrm{Hg}, \mathrm{HCO}_{3}{ }^{-2} 1 \mathrm{mEq} / \mathrm{L}, \mathrm{Na}^{+} 133 \mathrm{mEq} / \mathrm{L}$, $\mathrm{K}^{+} 2.8 \mathrm{mEq} / \mathrm{L}$, ionized $\mathrm{Ca}^{2+} 1.10$, and $\mathrm{Hb} 10.2 \mathrm{~g} / \mathrm{dL}$. Since our patient had laboratory value of serum $\mathrm{K}+$ to be $3.7 \mathrm{mEq} / \mathrm{L}$ (done 2 hours prior to surgery), we did not aggressively treat hypokalemia based on the $\mathrm{ABG}$ report.

Surgically, two burr-holes were made, anteriorly at the junction of superficial temporal line and coronal suture and posteriorly around the parietal eminence. First, the dura of the anterior burr-hole was opened in a cruciate manner and coagulated with bipolar diathermy. The collected blood (motor oil colored) in the subdural space was allowed to drain gradually. Thereafter, the posterior dura was incised and coagulated, and the subdural blood drained passively. Since the brain did not rise to the surface after spontaneous drainage of blood owing to septation and residual blood, an IFT of size 6 FG was inserted for approximately $5 \mathrm{~cm}$ through one of the burr-holes. The subdural space containing the residual blood was then irrigated with $0.9 \%$ normal saline injected through the port of the IFT. This maneuver enabled the drainage of the blood mixed with saline through the other burr-hole. Approximately, $1500 \mathrm{~mL}$ of saline was used for irrigation till the effluent was clear. However, immediately

\section{Published online}

September 12, 2020
DOI https://doi.org/

$10.1055 / \mathrm{s}-0039-1696080$

ISSN 2348-0548.
(C2020. Indian Society of Neuroanaesthesiology and Critical Care. This is an open access article published by Thieme under the terms of the Creative Commons Attribution-NonDerivative-NonCommercial-License, permitting copying and reproduction so long as the original work is given appropriate credit. Contents may not be used for commercial purposes, or adapted, remixed, transformed or built upon. (https://creativecommons.org/licenses/by-nc-nd/4.0/). Thieme Medical and Scientific Publishers Pvt. Ltd. A-12, 2nd Floor, Sector 2, Noida-201301 UP, India 
following irrigation, the surgeon noticed brain bulge and worsening of GCS to 9/15 (E2V2M5). It was not associated with any change in hemodynamic parameters. An ABG was done that was almost consistent with baseline values and $\mathrm{PCO}_{2}$ of $38 \mathrm{~mm} \mathrm{Hg}$. Intravenous mannitol $(100 \mathrm{~mL})$ was then administered over a period of 20 minutes. By this time, the surgery was completed, and the patient was shifted for urgent noncontrast computed tomography (NCCT) scan of brain that showed new onset ipsilateral IPH and pneumocephalus within the IPH cavity (suggesting irrigation injury) along with ipsilateral compression of lateral ventricle and midline shift ( - Fig. 1B). Retrospectively, the most probable cause of such devastation was ascertained to be the migration of IFT into the brain parenchyma during the process of irrigation. Immediately, tracheal intubation was done, and the patient was shifted to intensive care unit (ICU) for mechanical ventilation and other medical management. However, 6 hours post-surgery, the patient died due to uncal herniation.

\section{Case 2}

Another similar case, whereby a 76-year-old male patient (initial GCS-E3V4M5), who presented with right-sided hemiplegia, was operated under general anesthesia for left FTP acute on CSDH ( - Fig. 2A) with same surgical plan using IFT, by a different neurosurgeon. After the procedure, at the time of tracheal extubation, the patient manifested with worsening sensorium and persistence of right-sided hemiplegia and so it was decided to retain the endotracheal tube in situ. An urgent NCCT scan of brain was done that revealed new onset ipsilateral IPH and pneumocephalus within the IPH cavity along with ipsilateral compression of lateral ven-

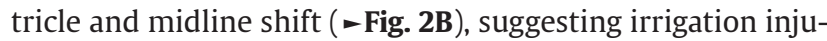
ry. The patient was mechanically ventilated and managed conservatively in ICU. On the fourth postoperative day, trachea was extubated following improvement in sensorium and the patient was finally discharged on 13th postoperative

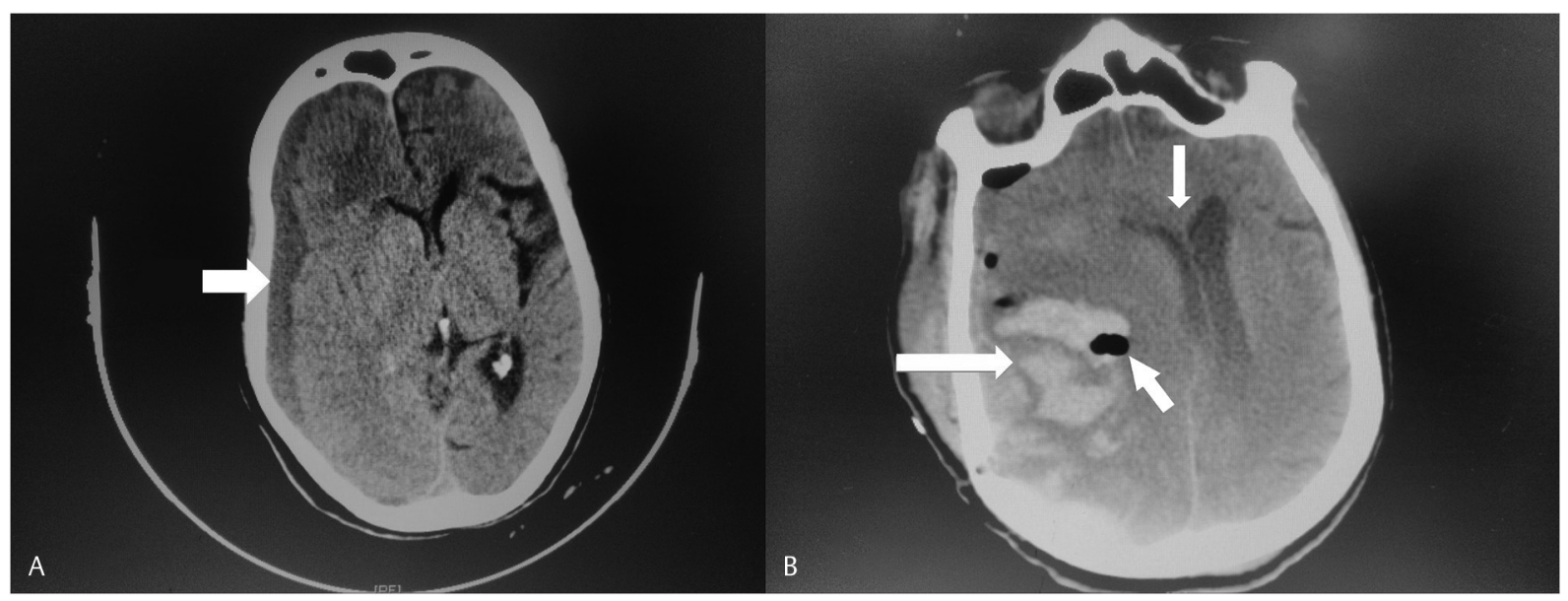

Fig. 1 Case 1, noncontrast computed tomography of the brain: (A) right frontotemporoparietal chronic subdural hematoma (arrow) and (B) right intraparenchymal hemorrhage (horizontal arrow) and pneumocephalus (oblique arrow) within the intraparenchymal hemorrhage cavity along with ipsilateral compression of lateral ventricle and midline shift (vertical arrow).

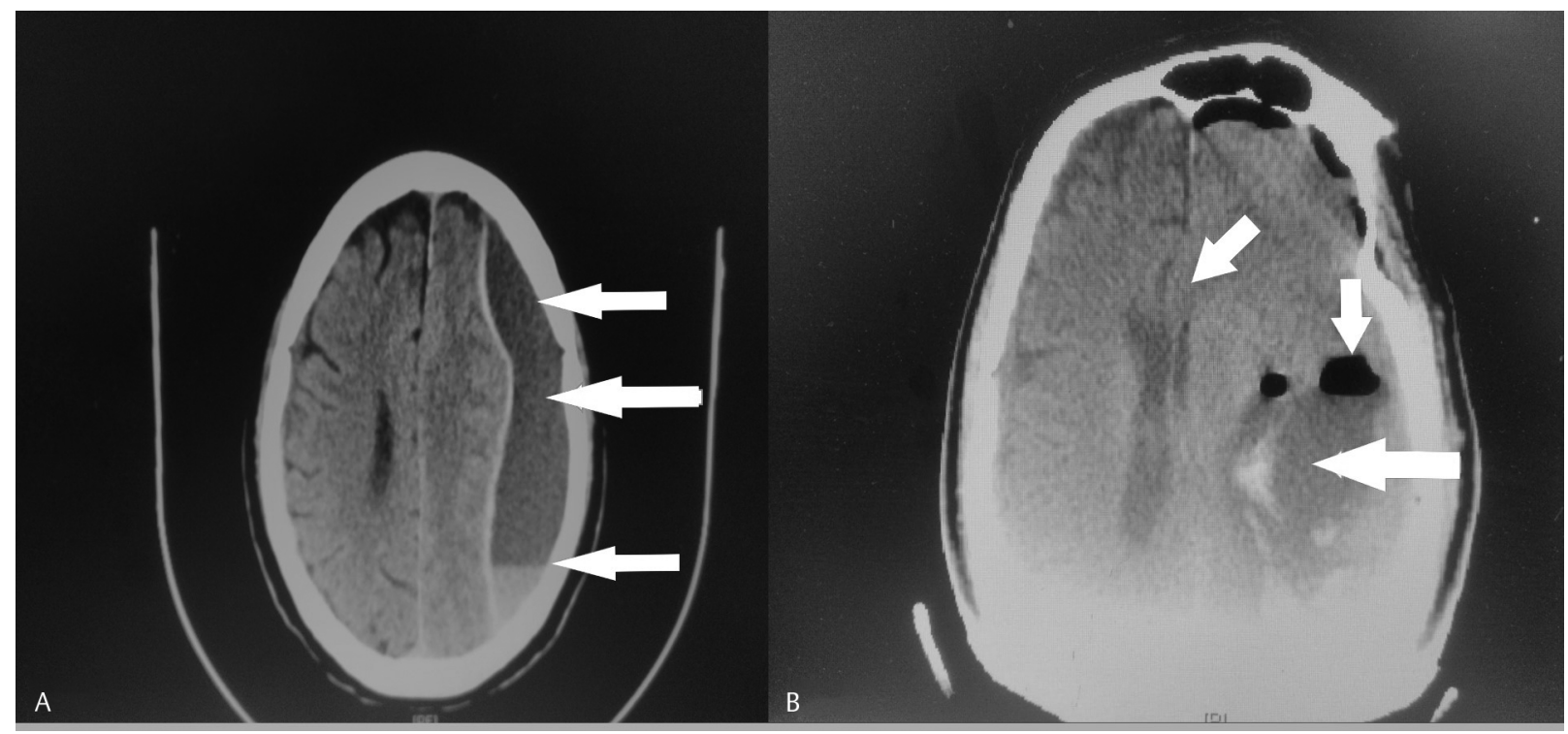

Fig. 2 Case 2, noncontrast computed tomography of the brain: (A) left frontotemporoparietal acute on chronic subdural hematoma (arrows) and (B) left intraparenchymal hemorrhage (horizontal arrow) and pneumocephalus (vertical arrow) within the intraparenchymal hemorrhage cavity along with ipsilateral compression of lateral ventricle and midline shift (oblique arrow). 
day with GCS of E3V5M5 but with persistence of right-sided hemiplegia.

\section{Discussion}

Various surgical approaches for symptomatic SDH have been described in literature that include twist-drill craniostomy, one or two burr-hole craniostomy, and craniotomy. ${ }^{2-4}$ Although rapid improvement in neurological functions is the hallmark of CSDH evacuation, the rate of complications irrespective of the type of surgical technique is high $(5-10 \%) .{ }^{5}$ Such complications include recurrence of hematoma, subdural empyema, tension hydrocephalus, seizures, intracerebral hematoma, and cerebral infarction. ${ }^{6}$ Even though rare, subarachnoid hemorrhage from aneurysmal bleeding following evacuation of CSDH has also been reported. ${ }^{7,8}$ The pathophysiologic mechanism of each of these complications may be different.

The most insidious complication following CSDH evacuation is intracerebral hemorrhage $(0.7-5 \%) .{ }^{9}$ Various hypotheses have been postulated for its causation. The most important underlying cause includes rapid decompression of hematoma and thus it is always recommended to allow gradual evacuation..$^{5,910}$ In both of our cases, two burr-hole craniostomy was done for hematoma evacuation. Gradual decompression was done (as described above) and thus intraparenchymal bleed in both our cases was unlikely due to this mechanism.

The most likely cause of IPH in our cases was due to the migration of IFT into the brain parenchyma as evident by the presence of air within the IPH cavity. Use of IFT for irrigating CSDH with septum is not an uncommon practice. Usual practice involves allowing initial passive drainage of blood (after incising dura) followed by irrigation with IFT if the brain does not rise to the surface (indicating residual hematoma). In our both cases, presence of septation and residual blood necessitated insertion of IFT. In addition, the burr-holes were relatively small ( $\sim 8-9 \mathrm{~mm}$ ), and thus the passage of IFT into a false tract could not be recognized initially. Only when a deterioration in neurological function was seen, a search was made to identify the most appropriate cause. In both cases, the CT scan showed almost identical presentation of IPH along with pneumocephalus within the IPH cavity. We also hypothesize that the volume and speed of irrigation fluid might have further contributed to the injury process.

Thus, even though IFT-guided CSDH evacuation is commonly practiced in presence of septum and residual blood, inherent risks associated with this maneuver should be kept in mind. Ideally, use of IFT should be avoided. Irrigation should be performed by directly instilling saline into the burr-hole till the point of clear effluent. Irrigation should be gentle, and the surgeon should be sure that there is no resistance and that the washing solution is not entrapped creating intracranial hypertension. However, if deemed necessary, a more flexible tube such as external ventricular catheter (without stylet) should be considered instead of rigid IFT. Further, it can be retained as a closed-drainage system after irrigation. Although burr-hole craniostomy is associated with a low recurrence rate and lesser complications, ${ }^{3,11}$ burr-hole size should be generous (at least $15 \mathrm{~mm}$ ) to ensure proper visualization of the irrigating tube.

Lastly, even if external ventricular catheter is not available and if residual hematoma needs to be drained, then IFT should be introduced very gradually by virtue of hydrodissection. A careful introduction of the "tube/drain" can be facilitated by a Bayonet forceps to avoid whiplash effects. The tube should be withdrawn even on slight resistance and redirected till correctly placed.

\section{Conclusion}

The IFT should be used with caution for irrigation of CSDH and should always be advanced gradually and gently without resistance. If deemed necessary, a safe alternative such as external ventricular drain should be looked for.

Conflict of Interest

None declared.

\section{References}

1 Prabhu SS, Zauner A, Bullock MR, Chronic Subdural Hematoma. In: Winn R, ed. Youmans Neurological Surgery. Philadelphia, PA: WB Saunders; 2003;5170-5171

2 Smith MD, Kishikova L, Norris JM. Surgical management of chronic subdural haematoma: one hole or two? Int J Surg 2012;10(9):450-452

3 Lega BC, Danish SF, Malhotra NR, Sonnad SS, Stein SC. Choosing the best operation for chronic subdural hematoma: a decision analysis. J Neurosurg 2010;113(3):615-621

4 Djoubairou BO, Onen J, Doleagbenou AK, El Fatemi N, Maaqili MR. Chronic subdural haematoma associated with pre-eclampsia: case report and review of the literature. Neurochirurgie 2014;60(1)(2):48-50

5 Pencalet P. Complications of chronic subdural hematoma in the adult [Article in French]. Neurochirurgie 2001;47(5):491-494

6 Mori K, Maeda M. Surgical treatment of chronic subdural hematoma in 500 consecutive cases: clinical characteristics, surgical outcome, complications, and recurrence rate. Neurol Med Chir (Tokyo) 2001;41(8):371-381

7 Miyazaki T, Matsumoto Y, Ohta F, Daisu M, Moritake K. A case of unknown origin subarachnoid hemorrhage immediately following drainage for chronic subdural hematoma. Kurume Med J 2004;51(2):163-167

8 Stefini R, Ghitti F, Bergomi R, Catenacci E, Latronico N, Mortini P. Uncommon presentation of ruptured intracranial aneurysm during surgical evacuation of chronic subdural hematoma: case report. Surg Neurol 2008;69(1):89-92, discussion 92

9 Ulivieri S, Oliveri G. Intracerebral haemorrhage following surgical evacuation of chronic subdural haematoma: case report. G Chir 2008;29(5):233-234

10 Gyu HA, Cheol SJ. "Controlateral" acute subdural and intracerebral hemorrhage occurring simultaneously after evacuation of huge chronic subdural hematoma. J Kor Neurotraumatol Soc 2011;7:39-42

11 Liu Y, Xia JZ, Wu AH, Wang YJ. Burr-hole craniotomy treating chronic subdural hematoma: a report of 398 cases. Chin J Traumatol 2010;13(5):265-269 